\title{
Optimización de la producción de lodos en un sistema de lodos activados a través de la calibración del modelo ASM1
}

\section{Optimization of the Production of Sludge in an Activated Sludge System Through the Calibration of the ASM1 Model}

\author{
Espinosa-Rodríguez M.A. \\ Programa de Ingeniería Química \\ Universidad Autónoma de Nayarit \\ Correo:mangelespinosa@hotmail.com
}

Fall C.

Centro Interamericano de Recursos del Agua

Universidad Autónoma del Estado de México

Correo:c-fa-ll@hotmail.com

Información del artículo: recibido: junio de 2013, reevaluado: agosto de 2013, aceptado: octubre de 2013

\section{Resumen}

Recientemente se han propuesto varios protocolos de calibración para el modelo ASM1 y cada uno de ellos hace énfasis en obtener un buen ajuste en la producción de lodos. Aquí es precisamente donde se evalúan las discrepancias y se hacen los reajustes de datos mediante un balance de materia. El objetivo de este trabajo fue realizar, primero, un balance de flujos de la planta de tratamiento de aguas residuales "Dulces Nombres" en Monterrey, Nuevo León, México, para luego ajustarlos hasta cerrar adecuadamente el balance de materia para fósforo total, sólidos suspendidos totales y sólidos suspendidos inorgánicos (ISS). Cabe señalar que para calibrar el modelo biológico fue importante determinar de manera confiable el caudal de purga de lodos $\left(\mathrm{Q}_{\mathrm{WAS}}\right)$, ya que de este depende el tiempo medio de retención celular $\left(\theta_{\mathrm{C}}\right)$ en los reactores, lo cual se realizó satisfactoriamente a través del balance de los ISS y utilizando el software GPS-X para la simulación de escenarios de optimización.

\footnotetext{
Abstract

Recently several protocols have been proposed for the calibration the ASM1 model and each one of them emphasizes obtaining a good adjustment in the production of sludge. Precisely here is where the discrepancies are evaluated and adjustments made to data, through a mass balance. The objective of this work was to conduct first a balance of flows in the wastewater treatment plant "Dulces Nombres" in Monterrey, Nuevo Leon, Mexico, and then adjust them until closing properly the mass balance for the total phosphorus, total suspended solids, and inorganic suspended solids (ISS). On might say that to calibrate the biological model was important to determine reliably the flow of sludge (QWAS) since on it depends the cell retention mean time $\left(\theta_{C}\right)$ in the reactor; this was accomplished successfully through the balance of the ISS and using of GPS-X software for the simulation of scenarios of optimization.
}

\section{Descriptores:}

- modelo ASM1

- calibración

- purga de lodos

- balance de flujos

- balance de materia
Keywords:

- ASM1 model

- calibration

- sludge purge

- flows balance

- mass balance 


\section{Introducción}

Los modelos de lodos activados (ASM), son metodologías avanzadas que representan el "estado de arte" aplicadas en procesos de lodos activados. Los modelos ASM1 y ASM3 son capaces de simular la remoción de materia orgánica, la nitrificación y desnitrificación, mientras que el ASM2 y ASM2d se utilizan cuando hay interés de simular además la remoción de fósforo (Henze et al., 2002). Sin embargo, estos modelos deben adaptarse y calibrarse para una planta de tratamiento de aguas residuales (PTAR) específica, ya que no se ha presentado en la literatura un procedimiento de calibración que sea adaptable en cualquier PTAR.

La calibración se puede definir como la adaptación de un modelo para describir cierta cantidad de información obtenida de la PTAR bajo estudio (Petersen et al., 2002). Desde la publicación del modelo ASM1 (Henze et al., 1987), no se ha definido todavía un procedimiento completo para su calibración, los reportes de la literatura así lo muestran (Petersen et al., 2002; Hulsbeek et al., 2002; WERF, 2003; Langergraber et al., 2004; Gürkan et al., 2005; Fall y Loaiza, 2007; Fall et al., 2011). Sin embargo, cada uno de ellos hace hincapié en obtener un buen ajuste en la producción de lodos, ya que es ahí donde se evalúan las discrepancias y se hacen los reajustes de datos a través de un balance de materia. El objetivo en la calibración no es alcanzar un ajuste perfecto, sino lograr que el modelo tenga capacidad de predicción dentro de un rango útil de aplicación (Henze et al., 2002).

El modelo ASM1, se presenta en notación matricial y se define por 13 componentes $(7$ de materia orgánica soluble y 6 particulados) y 8 procesos ( 3 de crecimiento, 2 de decaimiento y 3 de hidrólisis). De los 13 componentes, 7 se relacionan con materia orgánica del agua y lodo medidos como DQO (demanda química de oxígeno), 4 son constituyentes nitrogenados y se incluye además el oxígeno y la alcalinidad. El modelo utiliza la DQO como componente, ya que de esta forma se pueden realizar balances de masa; en este contexto, la materia orgánica se divide en DQO (biodegradable y no biodegradable) y biomasa (Henze et al., 1987).

La adaptación y calibración del modelo ASM1 para la planta "Dulces Nombres", se realizó con base en varios procedimientos: se combinó el protocolo de STOWA (Hulsbeek et al., 2002), el esquema propuesto por Petersen et al. (2002) y la secuencia de calibración propuesta por WERF (2003), con lo cual se obtuvo una metodología original. Se eligió el protocolo de STOWA (Hulsbeek et al., 2002), porque es el resultado de la experiencia acumulada en la calibración de más de 100 PTAR y las estra- tegias para la calibración de los parámetros cinéticos y estequiométricos del modelo ASM1 están bien definidas. Por otra parte, la integración de los diferentes estudios y pruebas realizadas en la planta, se basó en el protocolo de calibración de Petersen et al. (2002), el cual desarrolló un procedimiento sistemático de calibración para el modelo ASM1 evaluando plantas con alimentación combinada de agua residual (municipal-industrial), que es el caso de la planta "Dulces Nombres". Por último se utilizó la secuencia de calibración de WERF (2003), en particular, los procedimientos para el reajuste de flujos de purga, el cierre de balances de masa y el ajuste de los principales parámetros cinéticos y estequiométricos del modelo ASM1.

Para la calibración de la planta se realizaron estudios de:

a) caracterización del influente, efluente y lodos, incluyendo la determinación de las fracciones de la DQO y nitrógeno;

b) determinación del comportamiento hidráulico de los reactores a través de pruebas de trazador;

c) evaluación de las características de sedimentación del lodo a través de pruebas en columna;

d) campaña de monitoreo de gastos y

e) perfiles operacionales en los reactores (temperatura, oxígeno disuelto).

También se evaluaron las tasas de crecimiento y de decaimiento heterotrófico $\left(\mu_{\text {HMAX }} \mathrm{y} b_{\mathrm{H}}\right)$ y el coeficiente de rendimiento de la biomasa heterótrofa $\left(\mathrm{Y}_{\mathrm{H}}\right)$, con la meta de explorar su variación en función de la temperatura en el rango de 20 a $32^{\circ} \mathrm{C}$ (Espinosa et al., 2012).

En este contexto, el objetivo de este trabajo es presentar el proceso de calibración del modelo ASM1 aplicado en el sistema de lodos activados de la PTAR "Dulces Nombres" que llevaron a la optimización de la producción de lodos, para lo cual fue necesario determinar de manera confiable el caudal de purga de lodos $\left(Q_{\text {WAS }}\right)$ ya que de este depende el tiempo medio de retención celular $\left(\theta_{C}\right)$ en los reactores, lo que se realizó satisfactoriamente mediante balance de los sólidos suspendidos inorgánicos (ISS). Los resultados de los balances de flujo y de materia del sistema en conjunto con los resultados de otras pruebas, se alimentaron al software GPS-X con el cual se calibró el modelo ASM1 y se simularon los escenarios de optimización.

\section{Metodología}

El modelado de la PTAR se enfocó en el proceso de lodos activados (aireación y sedimentación secundaria). 
Este proceso (figura 1) inicia en la caja repartidora $\left(\mathrm{CR}_{2}\right)$ y comprende un tren de 5 reactores en paralelo $\left(R_{1}\right.$ a $\left.R_{5}\right)$ y 6 sedimentadores secundarios $\left(\mathrm{CS}_{1}\right.$ a $\left.\mathrm{CS}_{6}\right)$. Los 5 reactores descargan en una caja repartidora $\left(\mathrm{CR}_{3}\right)$ donde se distribuye el licor mezclado a los 6 sedimentadores secundarios. En este sistema de tratamiento de "Dulces Nombres", el reactor 5 y el clarificador secundario 2 se manejan de manera independiente. La purga de lodo de los 6 sedimentadores secundarios se lleva a cabo en la estación de bombeo de lodos (EBL) para luego enviarlos a tratamiento. En el caso de la recirculación de lodos, los clarificadores 1, 3, 4, 5, y 6 descargan en EBL para luego enviar el lodo a la estación de recirculación de lodos (ERL) y de aquí distribuirse en los primeros 4 reactores; la recirculación del $\mathrm{CS}_{2}$ se bombea de manera independiente al $\mathrm{R}_{5}$ también a través de ERL.

El modelado se realizó en dos líneas: la independiente (Reactor 5-Clarificador Secundario 2) y la conformada por los reactores 1 a 4 y sus clarificadores secundarios para la línea 2; para ello, fue necesario primeramente identificar todos los segmentos de la PTAR y por tanto todos los puntos posibles de medición de gastos, los cuales se muestran en la figura 2. En consecuencia, se diseñó el programa de medición de flujos y una campaña intensiva de muestreo.
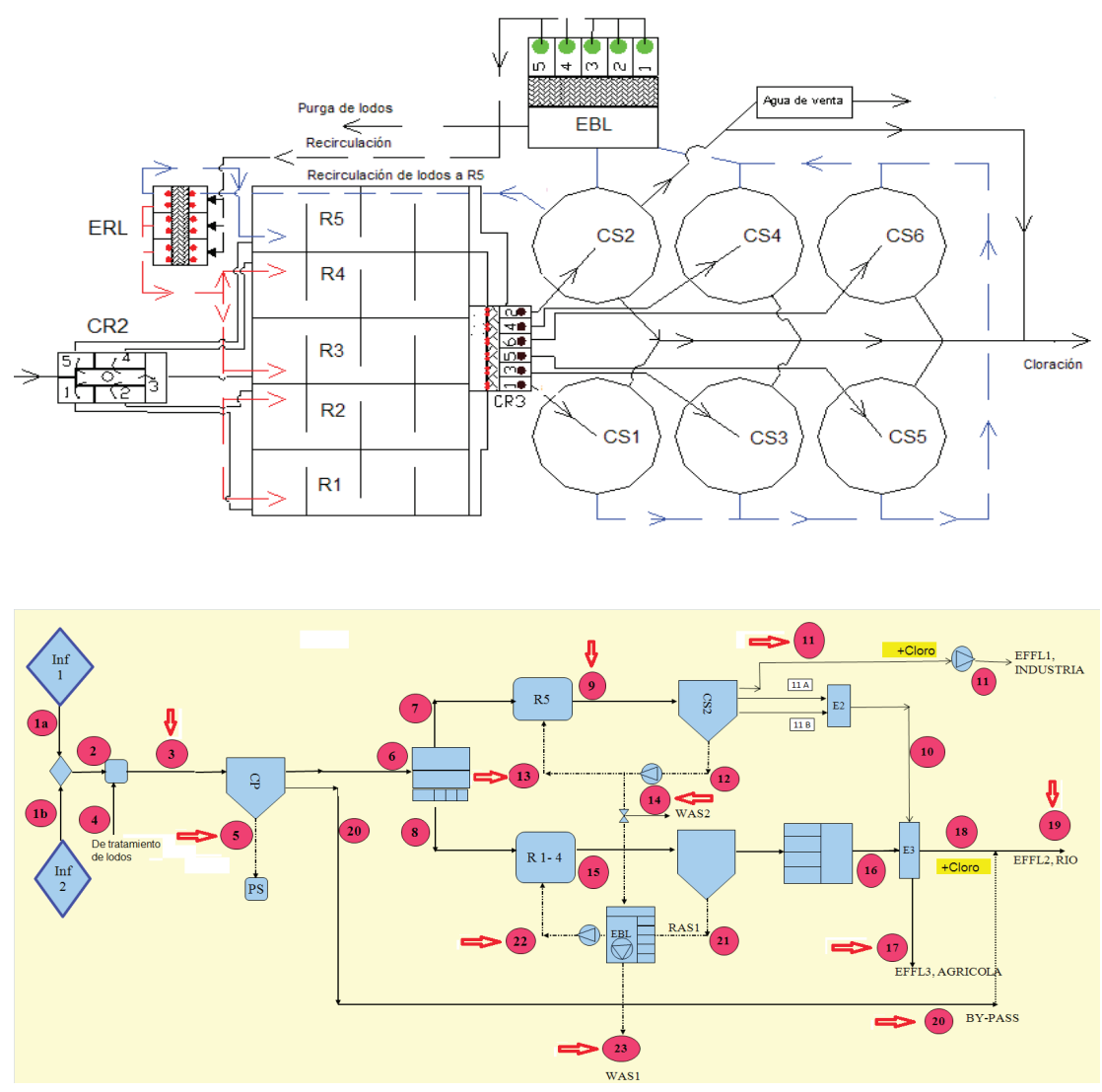

De acuerdo con la figura 2 se tomó la decisión de considerar como límite solamente el proceso de lodos activados, pero incluyendo para propósitos de información, algunos puntos de monitoreo alrededor de los sedimentadores primarios. Se identificaron 23 puntos de medición, sin embargo este número se redujo, ya que muchos de ellos se podrían obtener con un balance de flujos. Finalmente los puntos de medición mínimos a monitorear identificados fueron: 3, 5, 9, 11, 13, 14, 17, 19, 20, 22 y 23 (figura 2). El punto 19 se midió solamente para verificar algunos balances de masa. Adicionalmente, se tomó la decisión de verificar algunos gastos entre los reactores $R_{1}$ a $R_{4}$, y para ello se instalaron medidores de flujo en tres puntos a lo largo del canal de salida de los reactores; los puntos de medición identificados fueron: $15 \mathrm{a}, 15 \mathrm{~b}$ y $15 \mathrm{c}$.

Durante la campaña de medición de gastos, se utilizaron medidores doppler (tipo transit-time) y ultrasónicos. También se contó con el apoyo de un sistema maestro digital de captura de datos implantado en la planta, por lo que no fue necesario tomar lecturas manualmente en los puntos de medición. Con los puntos monitoreados, fue posible calcular mediante un balance los demás flujos, como se especifica en la tabla 1.
Figura 1. Sistema de lodos activados PTAR “Dulces Nombres"

Figura 2. Identificación de puntos de medición de flujos en todos los segmentos de la PTAR 


\begin{tabular}{|c|c|c|c|}
\hline $\begin{array}{l}\text { Gasto no } \\
\text { medido }\end{array}$ & Gasto medido & $\begin{array}{c}\text { Gasto no } \\
\text { medido }\end{array}$ & Gasto medido \\
\hline $\mathrm{Q}_{6}$ & $=\mathrm{Q}_{3}-\mathrm{Q}_{5}-\mathrm{Q}_{20}$ & $\mathrm{Q}_{15 \_ \text {R1out }}$ & $=\mathrm{Q}_{15 \mathrm{a}}$ \\
\hline $\mathrm{Q}_{10}$ & $=Q_{9}-Q_{12}-Q_{11}$ & $Q_{15 \text { R2out }}$ & $=Q_{15 b}-Q_{15 a}$ \\
\hline $\mathrm{Q}_{12}$ & $=Q_{13}-Q_{14}$ & $\mathrm{Q}_{15 \text { Rtout }}$ & $=Q_{15 c}$ \\
\hline$\hat{Q}_{9}$ & $=\mathrm{Q}_{10}+\mathrm{Q}_{11}+\mathrm{Q}_{12}$ & $\mathrm{Q}_{15 \text { R3out }}$ & $=Q_{15}-Q_{15 R 1 \text { out }}-Q_{15 R 2 \text { out }}-Q_{15 R \text { tou }}$ \\
\hline $\mathrm{Q}_{7}$ & $=Q_{9}-Q_{13}$ & $Q_{9}$ R5out & $=Q_{9}$ \\
\hline $\mathrm{Q}_{8}$ & $=\hat{Q}_{6}-\hat{Q}_{7}$ & $\mathrm{Q}_{\mathrm{R} \text { lin }}$ & $=\widehat{Q}_{\mathrm{R} 1 \text { out }}-(1 / 4) \mathrm{Q}_{22}$ \\
\hline$\hat{\mathrm{Q}}_{15}$ & $=\mathrm{Q}_{8}+\mathrm{Q}_{22}$ & $\widehat{Q}_{R 2 \text { in }}^{-k i m i n}$ & $=\mathrm{Q}_{\mathrm{R} 2 \text { out }}-(1 / 4) \mathrm{Q}_{22}$ \\
\hline $\mathrm{Q}_{21}$ & $=\mathrm{Q}_{22}+\mathrm{Q}_{23}-\mathrm{Q}_{14}$ & $\hat{Q}_{\text {R } 4 \text { in }}$ & $=Q_{\text {R4out }}-(1 / 4) Q_{22}$ \\
\hline $\mathrm{Q}_{16}$ & $=Q_{15}-Q_{21}$ & $\mathrm{Q}_{R} \mathrm{R}$ in & $=Q_{\mathrm{R} 3 \text { out }}-(1 / 4) \mathrm{Q}_{22}$ \\
\hline $\mathrm{Q}_{18}$ & $=Q_{10}+Q_{16}-Q_{17}$ & $Q_{-R 5 i n}$ & $=Q_{7}$ \\
\hline $\mathrm{Q}_{19}$ & $=Q_{18}+Q_{20}$ & & \\
\hline
\end{tabular}

Tabla 1. Método de cálculo de gastos no medidos
Con respecto a la campaña intensiva de muestreo, se seleccionaron puntos estratégicos de toma de muestras, con el fin de obtener la mayor cantidad de resultados que ayudaran a obtener una buena calibración del modelo, así como para realizar los balances de materia necesarios para evidenciar errores antes de la calibración. La localización de los puntos de muestreo durante la campaña intensiva se indica en la figura 3. La campaña intensiva de muestreo duró 7 días, tomando muestras compuestas durante 24 horas. Todas las muestras se prepararon siguiendo los estándares normales específicos y se analizaron siguiendo los procedimientos de métodos estándar (APHA, 1989). Derivado de los resultados obtenidos en la campaña de muestreo, se realizó el fraccionamiento de la DQO y el $\mathrm{N}$ con el modelo de influent advisor propuesto en el software GPS-X, con el fin de convertir los datos obtenidos en la campaña en un conjunto de fracciones de substrato compatibles con ASM (fracción orgánica).

Como se observa en la figura 3, se tomaron muestras en: influente crudo (influente después del desarenador, punto 3); lodo primario (muestra tomada en cárcamo de bombeo de lodos primarios, punto 5); efluente de la sedimentación primaria (muestra tomada en caja repar-

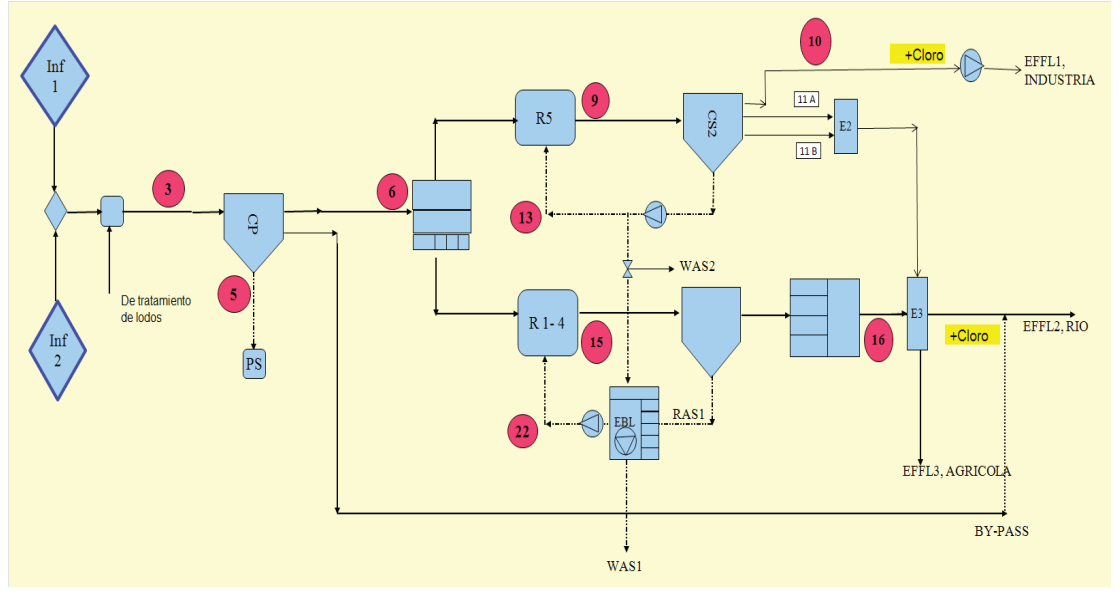

Figura 3. Localización de puntos de muestreo (PTAR Dulces Nombres) tidora que alimenta a reactores, punto 6); efluente del reactor 5 (muestra tomada en caja repartidora a clarificador secundario 2, punto 9); efluente del clarificador secundario 2 (muestra tomada en canal de CS2, punto 10); recirculación del reactor 5 (muestra tomada en estación de recirculación de lodos del $\mathrm{R}_{5}$, punto 13); efluente de $\mathrm{R}_{14}$ (muestra tomada en caja repartidora a CS1, punto 15); efluente de $\mathrm{CS}_{1,3,4,5,6}$ (muestra tomada en cárcamo de descarga sin cloro, punto 16) y, recirculación de $R_{1 \_4}$ (muestra tomada en estación de recirculación de lodos, punto 22).

Con los datos obtenidos en la campaña de medición de gastos y en la caracterización de la campaña intensiva de muestreo, se desarrollaron los balances de materia en una hoja de Excel. Para el balance de materia se desarrollaron 8 esquemas (figura 4) basados en el diagrama de flujo de la figura 2 . El procedimiento fue el siguiente:

a) Cierre del balance 1 (alrededor del clarificador primario), reajustando el flujo de lodos primarios $\left(Q_{5}\right)$.

b) Cierre del balance 3 (alrededor de la línea del reactor $\left.\mathrm{R}_{5}-\mathrm{CS}_{2}\right)$, reajustando el $\mathrm{Q}_{\mathrm{WAS}}\left(\mathrm{Q}_{14}\right)$. 
c) Cierre de los balances 2 y 6 (alrededor del tratamiento secundario total y alrededor de la línea del sistema $\left.R_{1-4}-C_{1-4}\right)$ reajustando el $Q_{W A S}\left(Q_{23}\right)$.

d) Cierre de los balances 4 y 5 (alrededor del $R_{5}$ y su clarificador $\mathrm{CS}_{2}$ solamente) reajustando $\mathrm{Q}_{7}$ (influente a $\mathrm{R}_{5}$ ).

e) Cierre de los balances 7 y 8 (alrededor de $R_{1-4}$ y su clarificador $\mathrm{CS}_{1,4}$ solamente), reajustando $\mathrm{Q}_{8}$ (influente a $\left.\mathrm{R}_{1-4}\right)$.

Considerando que para la calibración del modelo se requiere hacer un buen ajuste de lodos, se realizaron balances de masa para sólidos suspendidos totales (SST) y sólidos suspendidos inorgánicos (ISS). También se realizó el balance para el fósforo total $\left(\mathrm{P}_{\mathrm{TOT}}\right)$, ya que se considera la mejor base por removerlo a través del efluente y en la purga de lodo (Langergraber et al., 2004; Hulsbeek et al., 2002).

Para calibrar y simular la PTAR (en estado estacionario) con base en el modelo biológico ASM1 (Versión Mantis), se utilizó el software GPS-X. En este software, se pueden hacer simulaciones en estado estacionario y con condiciones dinámicas, por ello, es necesario elegir y especificar el modelo a utilizar en el simulador, según sea el requerimiento. Para la calibración del modelo de la planta "Dulces Nombres", se utilizaron los sub-modelos "COD fractions" para el influente, el "mantis" para los reactores y el modelo "simple $1 \mathrm{~d}$ " para los clarificadores secundarios. Sin embargo, en las etapas preliminares de verificación de los balances de SST, se llegó a utilizar el modelo "sludge" para representar el licor mezclado.

El proceso de alimentación de datos al software GPS$X$ fue el siguiente:

1) La calibración se inició con los parámetros por defecto del GPS-X (cinéticos, estequiométricos y de Arrhenius para ASM1); algunos de ellos se reajustaron posteriormente durante la calibración.

2) La caracterización y las relaciones estequiométricas básicas para el influente y las propiedades del lodo, se tomaron de la campaña de muestreo de 7 días.

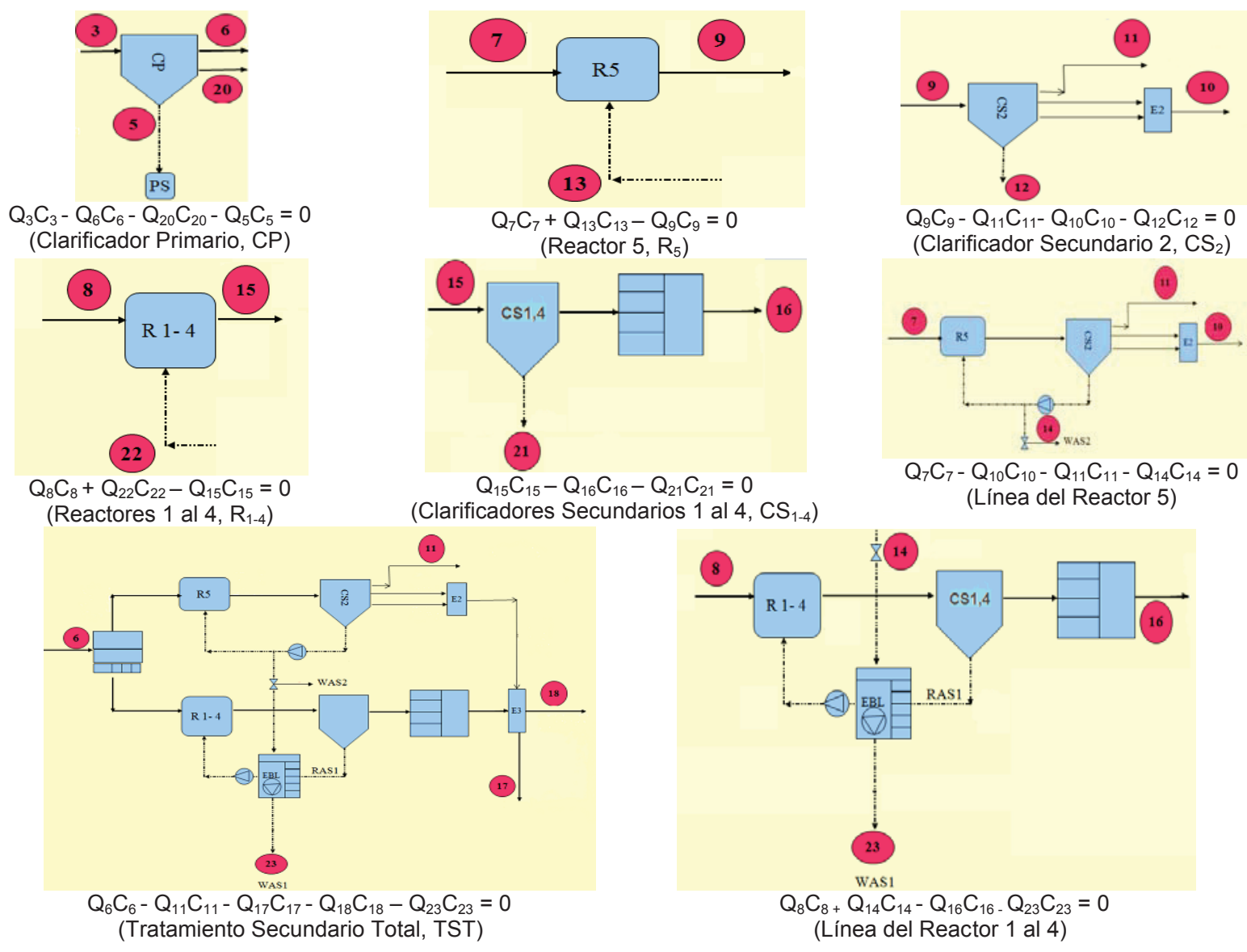

Figura 4. Balances de materia en puntos clave para la calibración del modelo ASM1 
3) Los datos de medición de flujos se especificaron en el simulador, tal como se midieron en campaña, aunque luego se modificaron en una etapa de reajuste para cerrar los balances de masa.

4) En la etapa de reajuste, estos gastos se modificaron con base en un balance de materia de los sólidos suspendidos inorgánicos (ISS = SST - SSV) en una hoja de Excel. Para la calibración del modelo biológico fue importante determinar de manera confiable el caudal de purga de lodos $Q_{\text {WAS, }}$ ya que de él depende $\theta_{C}$; esto se realizó mediante el balance de masa de ISS.

5) Los resultados del fraccionamiento de la $\mathrm{DQO}$ y el N se tomaron directamente del modelo "Influent Advisor".

6) Los perfiles operacionales de T y OD se aplicaron en el simulador según los resultados promedio obtenidos durante las campañas intensivas de muestreo.

7) El diagrama de flujo de la planta se conformó de acuerdo con la configuración de las tuberías, las dimensiones físicas de los tanques actuales y las del régimen hidráulico de los reactores.

8) Cada tanque de aireación se representó como una serie de reactores de mezcla completa, determinado con las pruebas de trazador y el modelado hidráulico.

9) Los resultados de los parámetros de Vesilind se tomaron del modelo de sedimentación (pruebas en columna) y otros datos para el caso de sedimentación se tomaron por defecto del software GPS-X para la calibración.

10) Por último, la calibración permitió comparar los valores medidos en campaña con los resultados arrojados por el software GPS-X. Con ello se obtuvo más información acerca de la operación de la planta y de parámetros técnicos que pueden ser utilizados en el modelo calibrado.

\section{Resultados y discusiones}

La tabla 2 muestra el resultado de los promedios diarios de los flujos obtenidos durante la campaña de monitoreo de gastos, así como los valores de los gastos no medidos que fueron calculados a través de un balance, con base en las ecuaciones de la tabla 1.

Un punto importante de la tabla 2 que debe resaltarse es los gastos de entrada a los primeros cuatro reactores $\left(Q_{R 1,23,4 i n}\right)$, ya que se supone que debería entrar un caudal igual en cada uno de ellos, como se detalla en el método de cálculo de la tabla 1, pero no sucede así en los reactores $\mathrm{R}_{3}$ y $\mathrm{R}_{4}$, en los cuales se detectaron valores incoherentes, mientras que los valores calculados para el $R_{1}$ y $R_{2}$ sí presentaron afinidad. Para estos últimos reactores, el flujo del licor mezclado se midió de manera independiente y los flujómetros operaron sin problemas. El flujómetro utilizado para la medición del licor mezclado del $\mathrm{R}_{4}$, presentó algunas deficiencias durante la campaña y en el caso de $R_{3}$, este se determinó con base en $Q_{15 \_ \text {R10ut' }} Q_{15 \_ \text {R2out }}$ y $Q_{15 \text { _RAout }}$ por lo que si la medición en $\mathrm{R}_{4}$ no es real, tampoco lo es para $\mathrm{R}_{3}$. En el caso del reactor $5\left(Q_{R 5 i n}\right)$ el flujo se calculó con base en la medición en $\mathrm{Q}_{9}$.

Los gastos de entrada a los reactores $R_{1}$ a $R_{5}$, se calcularon con base en $Q_{3}, Q_{5}$ y $Q_{20}$, como se desglosa en el balance de materia descrito en la tabla 1; por tanto, si el gasto del influente crudo $\left(Q_{3}\right)$ fue sobrestimado, o bien, el gasto en el by-pass del clarificador primario 4 $\left(Q_{20}\right)$ fue subestimado, que son los dos gastos con mayor incertidumbre, entonces, se puede generar el error en la partición de gastos a los reactores. La medición del flujo en el influente crudo $\left(Q_{3}\right)$, se realizó con un flujómetro de efecto doppler en una tubería excepcionalmente grande de diámetro $(2.59 \mathrm{~m})$, de ahí que resulte la sospecha de lecturas no reales en este punto, por otra parte, el gasto en el by-pass del clarificador primario $4\left(\mathrm{Q}_{20}\right)$ fue estimado usando un método basado en la inyección de trazador (Rhodamina WT), ya que era difícil monitorear con equipos de medición debido a la pendiente de la tubería.

Otro argumento favorable para la hipótesis de error en la medición de $\mathrm{Q}_{3}$, viene del historial de la PTAR, ya que en sus inicios se diseñó para operar con oxígeno puro, sin embargo, después se modificó con un sistema de aireación por difusores, con ello la capacidad de tratamiento resultó afectada. Los resultados del balance de materia mostrados posteriormente soportan esta hipótesis.

Tabla 2. Promedios diarios de gastos medidos y no medidos

\begin{tabular}{|c|c|c|c|c|c|}
\hline $\begin{array}{l}\text { Gasto } \\
\text { medido }\end{array}$ & $(\mathrm{L} / \mathrm{s})$ & $\begin{array}{l}\text { Gasto no } \\
\text { medido }\end{array}$ & $(\mathrm{L} / \mathrm{s})$ & $\begin{array}{l}\text { Gasto no } \\
\text { medido }\end{array}$ & $(\mathrm{L} / \mathrm{s})$ \\
\hline $\mathrm{Q}_{3}$ & 4216 & $\mathrm{Q}_{6}$ & 3824 & $\mathrm{Q}_{15 \_ \text {R1out }}$ & 1327 \\
\hline $\mathrm{Q}_{5}$ & 52 & $\mathrm{Q}_{10}$ & 834 & $\mathrm{Q}_{15 \text { R22out }}$ & 1325 \\
\hline $\mathrm{Q}_{11}$ & 185 & $\mathrm{Q}_{12}$ & 616 & $\mathrm{Q}_{15 \text { R R4out }}$ & 922 \\
\hline $\mathrm{Q}_{13}$ & 635 & $\mathrm{Q}_{9}$ & 1635 & $\mathrm{Q}_{15 \_ \text {R3out }}$ & 1988 \\
\hline$Q_{14}$ & 19 & $\mathrm{Q}_{7}$ & 1000 & $\mathrm{Q}_{9 \text { _R5out }}$ & 1635 \\
\hline $\mathrm{Q}_{17}$ & 292 & $\mathrm{Q}_{8}$ & 2824 & & \\
\hline $\mathrm{Q}_{19}$ & 3699 & $\mathrm{Q}_{15}$ & 5562 & & \\
\hline $\mathrm{Q}_{20}$ & 340 & $\mathrm{Q}_{21}$ & 2745 & & \\
\hline $\mathrm{Q}_{22}$ & 2738 & $\mathrm{Q}_{16}$ & 2817 & & \\
\hline $\mathrm{Q}_{23}$ & 26 & $\mathrm{Q}_{18}$ & 3359 & & \\
\hline$Q_{11 A}$ & 198 & $\mathrm{Q}_{19}$ & 3699 & & \\
\hline $\mathrm{Q}_{11 \mathrm{~B}}$ & 169 & $\mathrm{Q}_{-\mathrm{R} 1 \mathrm{in}}$ & 643 & & \\
\hline$Q_{15 a}$ & 1327 & $\mathrm{Q}_{\mathrm{R} 2 \mathrm{in}}^{-}$ & 641 & & \\
\hline $\mathrm{Q}_{15 \mathrm{~b}}$ & 2652 & $\mathrm{Q}_{\mathrm{R} 4 \mathrm{in}}$ & 238 & & \\
\hline \multirow[t]{2}{*}{$\mathrm{Q}_{15 \mathrm{c}}$} & 922 & $\mathrm{Q}_{-\mathrm{R} 3 \mathrm{in}}$ & 1304 & & \\
\hline & & $\mathrm{Q}_{\mathrm{R} \text { 5in }}^{-}$ & 1000 & & \\
\hline
\end{tabular}


Para efecto del balance de materia, los resultados de caracterización de fósforo total $\left(\mathrm{P}_{\text {TOTAL }}\right)$, sólidos suspendidos totales (SST) y sólidos suspendidos volátiles (SSV) durante la campaña intensiva de muestreo de 24 horas en 7 días, se describen en la tabla 3. Para el caso de los sólidos suspendidos inorgánicos (ISS), estos se obtuvieron con la diferencia entre los SST menos los SSV.

Con los resultados de medición de flujos y la caracterización mostrada en la tabla 3, se realizaron los balances de materia. Como se especifica y se recomienda para cerrar los balances totales se utilizaron los flujos de purga $Q_{5}, Q_{14}$ y $Q_{23}$. Las buenas prácticas de modelado sugieren que la calibración no se ajusta mientras que los balances de materia no estén cerrados adecuadamente (Meijer et al., 2002; WERF, 2003). En la tabla 4 se muestran los resultados obtenidos del balance de materia para $\mathrm{P}_{\mathrm{TOT}}$ SST e ISS de acuerdo con la hoja de Excel.

Para cerrar los balances presentados en la tabla 4, fue necesario el reajuste del flujo de la purga de clarificadores primarios $\left(\mathrm{Q}_{5}\right)$, de la purga de lodos $\left(\mathrm{Q}_{\text {was }}\right)$ del $\mathrm{CS}_{2}\left(\mathrm{Q}_{14}\right)$ y de la purga de lodos $\left(\mathrm{Q}_{\text {was }}\right)$ de los $\mathrm{CS}_{1,4}\left(\mathrm{Q}_{23}\right)$. $\mathrm{Al}$ variar estos gastos, se modificaron los flujos medidos en campaña $Q_{3}, Q_{9}, Q_{19}$ y los demás flujos incluyentes en el balance de materia según la tabla 1, entre ellos los flujos de influente a reactores $\left(Q_{7}\right.$ y $\left.Q_{8}\right)$.

Cabe mencionar que para la medición de flujos en la recirculación de lodos $Q_{13}$ y $Q_{22}$ (RAS), se contó con el equipo de medición de la planta y los instalados durante la campaña. Los resultados en ambos flujómetros fueron muy similares, por tanto, estos flujos no se modificaron en el balance de materia.

Otros gastos que no fueron reajustados fueron $\mathrm{Q}_{11} \mathrm{y}$ $\mathrm{Q}_{17}$. El primer flujo (agua de venta industrial) se tenía monitoreado en la planta durante la campaña y en la empresa que reutiliza el agua para la generación de energía eléctrica, en ambos casos los resultados de medición fueron similares. En el caso de $\mathrm{Q}_{17}$ (uso agrícola), la medición se realizó en un canal de concreto abierto para lo cual se tenían los datos necesarios para medir el flujo con un equipo ultrasónico; este equipo no presentó fallas y los datos fueron constantes.

Para $Q_{20}$, se determinó no reajustarlo, aun sabiendo que era un flujo medido con pruebas de trazador, de todas maneras, se decidió realizar los cálculos correspondientes sabiendo de antemano que el flujo del influente a la planta $\left(\mathrm{Q}_{3}\right)$ se modificaría. Se consideró para ello que el valor obtenido en $Q_{20}$ resultaba pequeño comparado al flujo $Q_{3}$. En la tabla 5 se muestran los flujos utilizados para el balance de materia y el reajuste de alguno de ellos. Los flujos mostrados en la columna de reajuste, se usaron para la calibración del modelo ASM1.
Aun cuando la hoja de Excel se diseñó para lograr los mejores consensos a fin de concluir los balances para los ISS, SST у $\mathrm{P}_{\text {тот }}$ para este proyecto, la prioridad fue el balance para los ISS. Los laboratorios instalados en las PTAR en México, normalmente tienen experiencia en la determinación de sólidos, pero no tanto para fósforo. Meijer et al., (2002), menciona que es recomendable examinar y resolver las discrepancias de los datos crudos antes de la calibración, lo cual es un medio de verificación y reajuste.

Para poder cerrar los balances de $\mathrm{P}_{\text {TOт }}$ con $(<17.2 \%$ de error), SST con $(<3.3 \%$ de error $)$ e ISS con $(<1.1 \%$ de error) según la tabla 4 , fue necesario disminuir los flujos de influente a los reactores $\left(\mathrm{Q}_{7}\right.$ y $\left.\mathrm{Q}_{8}\right)$. Se optó por hacer los ajustes (cierre de balance de materia según figura 4) de la mejor manera con base primero en los SSI, tratando de cerrar lo mejor posible los balances de SST y $\mathrm{P}_{\text {тотаL }}$ y que no se fueran a los extremos, lo cual se obtuvo para SST, pero no muy satisfactoriamente para el fósforo; la posible causa podría ser los resultados obtenidos en laboratorio para el caso del fósforo (no hay mucha práctica en su determinación); no así para los resultados de sólidos en donde se tiene bastante experiencia. También pudo haber ocurrido una probable desestabilización del pseudo estado estable por el incidente ocurrido una semana antes de la campaña de muestreo, donde los lodos en la sedimentación primaria se derramaron por la superficie de los tanques. De acuerdo con Langergraber et al., (2004), los balances de materia deben cerrar con una incertidumbre no mayor que $5 \%$ para el caso del $\mathrm{P}_{\text {TOTAL }}$ y $10 \%$ para SST, lo cual se cumplió para SST $(<3.3 \%)$ y no para $\mathrm{P}_{\text {TOTAL }}(<17.2 \%)$ en el caso de la planta "Dulces Nombres".

Con los resultados obtenidos en el balance de materia, la calibración del modelo ASM1 para la PTAR “Dulces Nombres" se realizó en 10 pasos secuenciales (Fall et al., 2011) con ayuda del software GPS-X. El primer paso involucró el balance de ISS en las líneas del $R_{5} y$ $\mathrm{R}_{1-4}$ ajustando el flujo de purga $\left(\mathrm{Q}_{\mathrm{WAS}}\right)$; el segundo paso fue desarrollar un balance de materia de SST en clarificadores $\left(\mathrm{CS}_{2}\right.$ y $\left.\mathrm{CS}_{1-4}\right)$ ajustando el flujo de recirculación $\left(Q_{\text {RAS }}\right)$ y el flujo del influente a los clarificadores; en el paso 3 se calibró la producción de lodos ajustando la concentración de sólidos en el licor mezclado de los reactores, utilizando la fracción de sustrato de la DQO particulada (frxs) como parámetro de ajuste. El paso 4 se utilizó para afinar (reajustar) las etapas 1, 2 y 3. El paso 5 fue básicamente la calibración de los SST en el efluente de los clarificadores secundarios mediante los parámetros de Vesilind (máxima velocidad de sedimentación y exponente de Vesilind) obtenidos en las pruebas de sedimentación en columna, mientras que en 
DOI: https://doi.org/10.1016/S1405-7743(15)72110-4

Optimización de la producción de lodos en un sistema de lodos activados a través de la calibración del modelo ASM1

Tabla 3. Caracterización de muestras compuestas (7 días) en la PTAR "Dulces Nombres"

\begin{tabular}{cccccccccc}
\hline & \multicolumn{7}{c}{$\begin{array}{c}\text { Puntos de muestreo } \\
\text { (concentraciones promedio) }\end{array}$} \\
\hline Parámetros $\left(\mathrm{g} / \mathrm{m}^{3}\right)$ & 3 & 5 & 6 & 9 & 15 & 13 & 22 & 10 & 16 \\
\hline SST & 501.0 & 36000.0 & 107.0 & 4156.0 & 4318.0 & 7790.0 & 7543.0 & 14.6 & 11.5 \\
SSV & 358.0 & 25737.0 & 82.0 & 3193.0 & 3305.0 & 5943.0 & 5781.0 & 11.6 & 8.5 \\
ISS & 143.0 & 10263.0 & 25.0 & 963.0 & 1013.0 & 1847.0 & 1762.0 & 3.0 & 3.0 \\
$P_{\text {TOTAL }}$ & 17.5 & 430.0 & 11.7 & 116.4 & 122.0 & 180.0 & 186.0 & 8.7 & 8.9 \\
\hline
\end{tabular}

Tabla 4. Resultados del balance de materia para $\mathrm{P}_{\text {TOT }}$, SST e ISS

\begin{tabular}{|c|c|c|c|c|c|c|c|c|}
\hline \multicolumn{9}{|c|}{ Balance de materia para el fósforo total $\left(\mathrm{P}_{\mathrm{TOT}}\right)$} \\
\hline & $\mathrm{CP}$ & R5 & CS2 & R1-4 & CS1-4 & LíneaR5 & T.S. T. & Línea R1-4 \\
\hline Entradas (kg/d) & 4627 & 10467 & 12269 & 46124 & 50996 & 591 & 2714 & 2232 \\
\hline Salidas (kg/d) & -4358 & -12269 & -10212 & -50996 & -46021 & -554 & -2585 & -2133 \\
\hline Diferencia & 268 & -1803 & 2058 & -4873 & 4975 & 38 & 129 & 99 \\
\hline$\%$ & 5.8 & -17.2 & 16.8 & -10.6 & 9.8 & 6.3 & 4.8 & 4.4 \\
\hline \multicolumn{9}{|c|}{ Balance de materia para sólidos suspendidos totales (SST) } \\
\hline Entradas (kg/d) & 132456 & 432799 & 438076 & 1803810 & 1804938 & 5408 & 24822 & 24125 \\
\hline Salidas $(\mathrm{kg} / \mathrm{d})$ & -136830 & -438076 & -423426 & -1804938 & -1803727 & -5458 & -24530 & -23893 \\
\hline Diferencia & -4373 & -5277 & 14650 & -1128 & 1211 & -50 & 292 & 233 \\
\hline$\%$ & -3.3 & -1.2 & 3.3 & -0.1 & 0.1 & -0.9 & 1.2 & 1.0 \\
\hline \multicolumn{9}{|c|}{ Balance de materia para sólidos suspendidos inorgánicos (ISS) } \\
\hline Entradas $(\mathrm{kg} / \mathrm{d})$ & 37807 & 102597 & 101508 & 421360 & 423437 & 1264 & 5800 & 5653 \\
\hline Salidas (kg/d) & -37569 & -101508 & -100370 & -423437 & -421396 & -1271 & -5791 & -5637 \\
\hline Diferencia & 238 & 1090 & 1138 & -2077 & 2041 & -7 & 9 & 16 \\
\hline$\%$ & 0.6 & 1.1 & 1.1 & -0.5 & 0.5 & -0.5 & 0.2 & 0.3 \\
\hline
\end{tabular}

Tabla 5. Resultados de flujos utilizados para el balance de materia de $\mathrm{P}_{\text {TOT }}$, SST e ISS

\begin{tabular}{|c|c|c|c|c|c|}
\hline Ecuación & Flujo & $\mathrm{Q}_{\text {inicial }}(\mathrm{L} / \mathrm{s})$ & Reajuste (L/s) & & $\mathrm{Q}\left(\mathrm{m}^{3} / \mathrm{d}\right)$ \\
\hline \multirow[t]{2}{*}{$\mathrm{Q}_{6}+\mathrm{Q}_{5}+\mathrm{Q}_{20}$} & $\mathrm{Q}_{3}$ & 4216 & 3060 & & 264384 \\
\hline & $\mathrm{Q}_{5}$ & 52 & 35 & & 3024 \\
\hline \multirow[t]{8}{*}{$\mathrm{Q}_{9}-\mathrm{Q}_{13}$} & $\mathrm{Q}_{7}$ & 1000 & 585 & & 50544 \\
\hline & $\mathrm{Q}_{11}$ & 185 & 185 & Rec/Qin & 15984 \\
\hline & $\mathrm{Q}_{13}$ & 635 & 635 & 1.08547 & 54864 \\
\hline & $\mathrm{Q}_{14}$ & 19 & 7 & & 605 \\
\hline & $\mathrm{Q}_{20}$ & 340 & 340 & & 29376 \\
\hline & $\mathrm{Q}_{22}$ & 2738 & 2738 & 1.30381 & 236563 \\
\hline & $\mathrm{Q}_{23}$ & 26 & 34 & & 2894 \\
\hline & $\mathrm{Q}_{17}$ & 292 & 292 & & 25229 \\
\hline $\mathrm{Q}_{8}+\mathrm{Q}_{7}$ & $\mathrm{Q}_{6}$ & 3824 & 2685 & & 231984 \\
\hline $\mathrm{Q}_{6}-\mathrm{Q} 7$ & $\mathrm{Q}_{8}$ & 2824 & 2100 & & 181440 \\
\hline $\mathrm{Q}_{8 / 4}$ & $\mathrm{Q}_{\text {inR1 }}$ & 706 & 525 & & 45360 \\
\hline $\mathrm{Q}_{7}+\mathrm{Q}_{13}$ & $\mathrm{Q}_{9}$ & 1635 & 1220 & & 105408 \\
\hline $\mathrm{Q}_{8}+\mathrm{Q}_{22}$ & $\mathrm{Q}_{15}$ & 5562 & 4838 & & 418003 \\
\hline $\mathrm{Q}_{13}-\mathrm{Q}_{14}$ & $\mathrm{Q}_{12}$ & 616 & 628 & & 54259 \\
\hline $\mathrm{Q}_{22}+\mathrm{Q}_{23}-\mathrm{Q}_{14}$ & $\mathrm{Q}_{21}$ & 2745 & 2765 & & 238853 \\
\hline$Q_{9}-Q_{12}-Q_{11}$ & $\mathrm{Q}_{10}$ & 834 & 407 & & 35165 \\
\hline $\mathrm{Q}_{15}-\mathrm{Q}_{21}$ & $\mathrm{Q}_{16}$ & 2817 & 2074 & & 179150 \\
\hline $\mathrm{Q}_{10}+\mathrm{Q}_{16}-\mathrm{Q}_{17}$ & $\mathrm{Q}_{18}$ & 3359 & 2189 & & 189086 \\
\hline $\mathrm{Q}_{18}+\mathrm{Q}_{20}$ & $\mathrm{Q}_{19}$ & 3699 & 2529 & & 218462 \\
\hline
\end{tabular}


el paso 6, se calibró la concentración de DQO en el efluente de la sedimentación secundaria ajustando la fracción inerte de la DQO soluble (frsi). En la etapa 7 se calibró el proceso de nitrificación en los reactores a través del balance del nitrógeno amoniacal ajustando la tasa máxima de crecimiento autotrófico $\left(\mu_{\mathrm{A}}\right)$, mientras que en el paso 8 se calibró la desnitrificación también en los reactores con la concentración de nitratos en el efluente como parámetro de respuesta y tomando como parámetro de ajuste el coeficiente medio de saturación de oxígeno $\left(\mathrm{K}_{\mathrm{O} 2 \text {,anox }}\right)$; los puntos de ajuste de ambos finalmente se realizó en el efluente de clarificadores secundarios. El paso 9 involucró la calibración de la desnitrificación, pero ahora en los clarificadores secundarios considerando la concentración de nitratos en el RAS. Por último el paso 10 involucró iteraciones para calibrar la PTAR total.

En las figuras 5 y 6 se presentan los escenarios de calibración para el balance de ISS en la línea del $\mathrm{R}_{5-} \mathrm{CS}_{2}$ y $R_{1,4-} C_{1,4}$ respectivamente, ajustando de nueva cuenta el $Q_{\text {WAS }}\left(Q_{14}\right.$ y $Q_{23}$ respectivamente).

Como se observa en la figura 5, el flujo de purga de lodos $\mathrm{Q}_{14}$ se modificó al utilizar el software GPS-X, pasando de $605 \mathrm{~m}^{3} / \mathrm{d}$ a $592 \mathrm{~m}^{3} / \mathrm{d}$, con ello quedaron calibrados los ISS en el $R_{5}\left(Q_{9}\right)$ en $962.2 \mathrm{~g} / \mathrm{m}^{3}$, que comparado con el resultado obtenido en campaña (963 $\mathrm{g} / \mathrm{m}^{3}$ ) es insignificante la diferencia. Por otra parte el flujo de purga de lodos $Q_{23}$ pasó de $2894 \mathrm{~m}^{3} / \mathrm{d}$ a 2200 $\mathrm{m}^{3} / \mathrm{d}$, según la figura 6 , quedando calibrados los ISS en el $R_{1,4}\left(Q_{15}\right)$ en $1002 \mathrm{~g} / \mathrm{m}^{3}$ muy similar al obtenido en la campaña de muestreo de $1013 \mathrm{~g} / \mathrm{m}^{3}$.

Ya ajustados los flujos de purga de lodos y calibrados los ISS correspondientes, se realizó el balance de SST alrededor de los clarificadores con lo cual se ajustaron los flujos de recirculación del $\mathrm{R}_{5}\left(\mathrm{Q}_{13}\right)$ y $\mathrm{R}_{1,4}\left(\mathrm{Q}_{22}\right) \mathrm{y}$, por tanto, los flujos del influente a $\mathrm{CS}_{2}\left(\mathrm{Q}_{9}\right)$ y $\mathrm{CS}_{1,4}\left(\mathrm{Q}_{15}\right)$, como se muestra en las figuras 7 y 8 . La etapa de reajuste para el cierre de balances de materia, es aceptada en ASM1, ya que partiendo de que los datos de laboratorio son generalmente correctos, aun así existe una probabilidad de error sobre las mediciones en la purga de lodos, por lo que se sugiere siempre verificarlo. Asimismo, la práctica de reajustar $\mathrm{Q}_{\mathrm{WAS}}$ se acepta sin mayor discusión, ya que el ajuste de flujos de purga es una práctica común en el modelado de PTAR (Meijer et al., 2002).

Como se observa en la figura 7 el flujo de recirculación $\mathrm{Q}_{13}$ se modificó con el software GPS-X. Para este caso el flujo de recirculación pasó de $54864 \mathrm{~m}^{3} / \mathrm{d}$ a $56364 \mathrm{~m}^{3} / \mathrm{d}$. Debido a este reajuste, el flujo del influente al $\mathrm{CS}_{2}\left(\mathrm{Q}_{9}\right)$ también se modificó y pasó de $105408 \mathrm{~m}^{3} / \mathrm{d}$ a 106908 $\mathrm{m}^{3} /$ d. Con estos reajustes se logró la calibración de los SST en el RAS $\left(\mathrm{Q}_{13}\right)$ quedando en $7792 \mathrm{~g} / \mathrm{m}^{3}$, el cual es muy similar al obtenido en la campaña de muestreo que fue de $7790 \mathrm{~g} / \mathrm{m}^{3}$. Por otra parte, en la figura 8, el flujo de recirculación $\mathrm{Q}_{22}$, se modificó al utilizar el software GPSX. Para este caso, el flujo de recirculación pasó de 236563 $\mathrm{m}^{3} / \mathrm{d}$ a $237163 \mathrm{~m}^{3} / \mathrm{d}$, debido a este reajuste, el flujo del influente al $\mathrm{CS}_{1,4}\left(\mathrm{Q}_{15}\right)$ también se modificó y pasó de $418003 \mathrm{~m}^{3} / \mathrm{d}$ a $418603 \mathrm{~m}^{3} / \mathrm{d}$. Con estos reajustes se logró la calibración de los SST en el RAS $\left(\mathrm{Q}_{22}\right)$ quedando en $7545 \mathrm{~g} / \mathrm{m}^{3}$ cuya diferencia con el obtenido en la campaña de muestreo $\left(7543 \mathrm{~g} / \mathrm{m}^{3}\right)$, es mínima.

Los cuatro escenarios de calibración mostrados, fueron la base para la simulación de escenarios de optimización. En la figura 9 se muestra el escenario de calibración y optimización final de la producción de lodos de la PTAR "Dulces Nombres".

Como se observa en la figura 9, finalmente el flujo de purga de lodos $Q_{14}$ quedó optimizado en $570 \mathrm{~m}^{3} / \mathrm{d}$ y el flujo de purga de lodos $Q_{23}$ en $2100 \mathrm{~m}^{3} / \mathrm{d}$. Los flujos

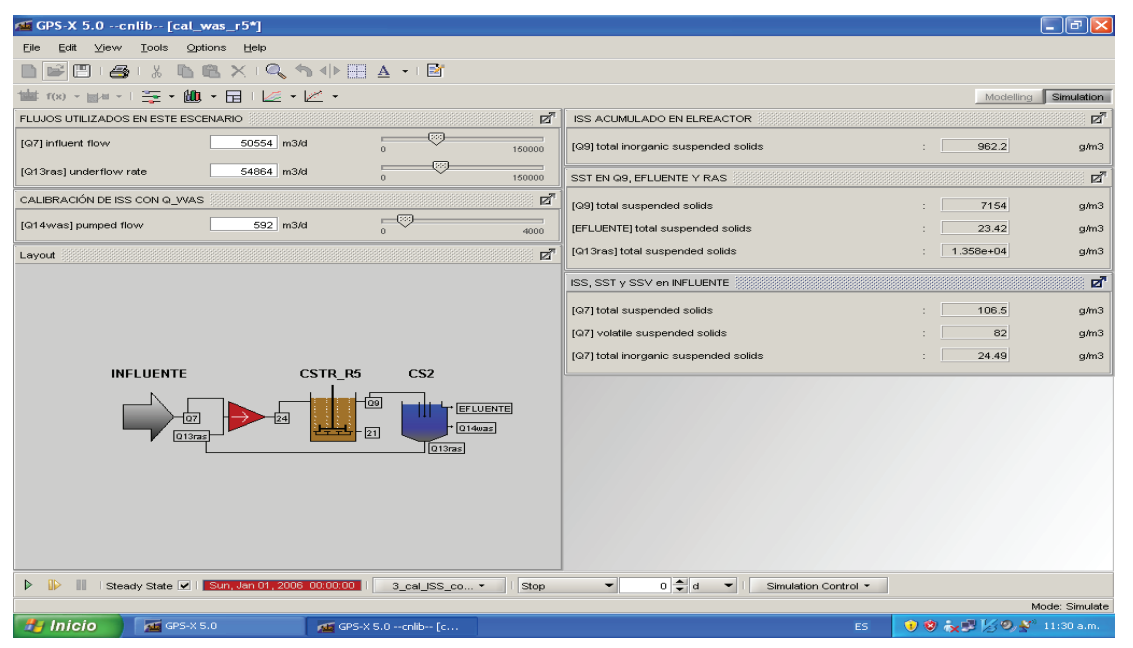

Figura 5. Calibración de ISS en línea de $\mathrm{R}_{5} \mathrm{CS}_{2}$. Escenario de simulación con GPS-X 

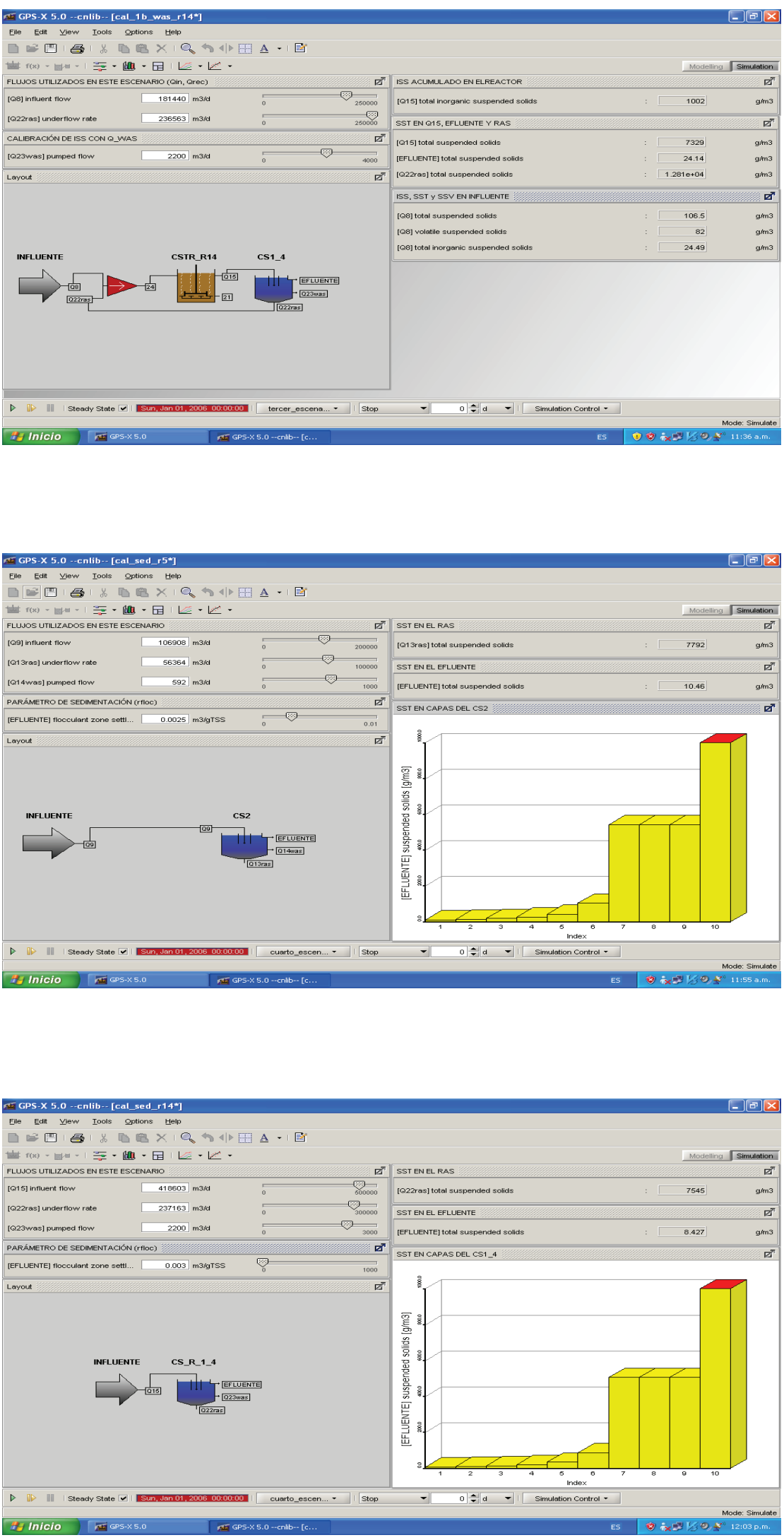

Figura 6. Calibración de ISS en línea de $\mathrm{R}_{1,4-} \mathrm{CS}_{1,4-4}$ Escenario de simulación con GPS-X
Figura 7. Calibración de SST en $\mathrm{CS}_{2}$ del $\mathrm{R}_{5}$ Escenario de simulación con GPS-X
Figura 8. Calibración de SST en $\mathrm{CS}_{1,4}$ del $\mathrm{R}_{1,4}$. Escenario de simulación con GPS-X 


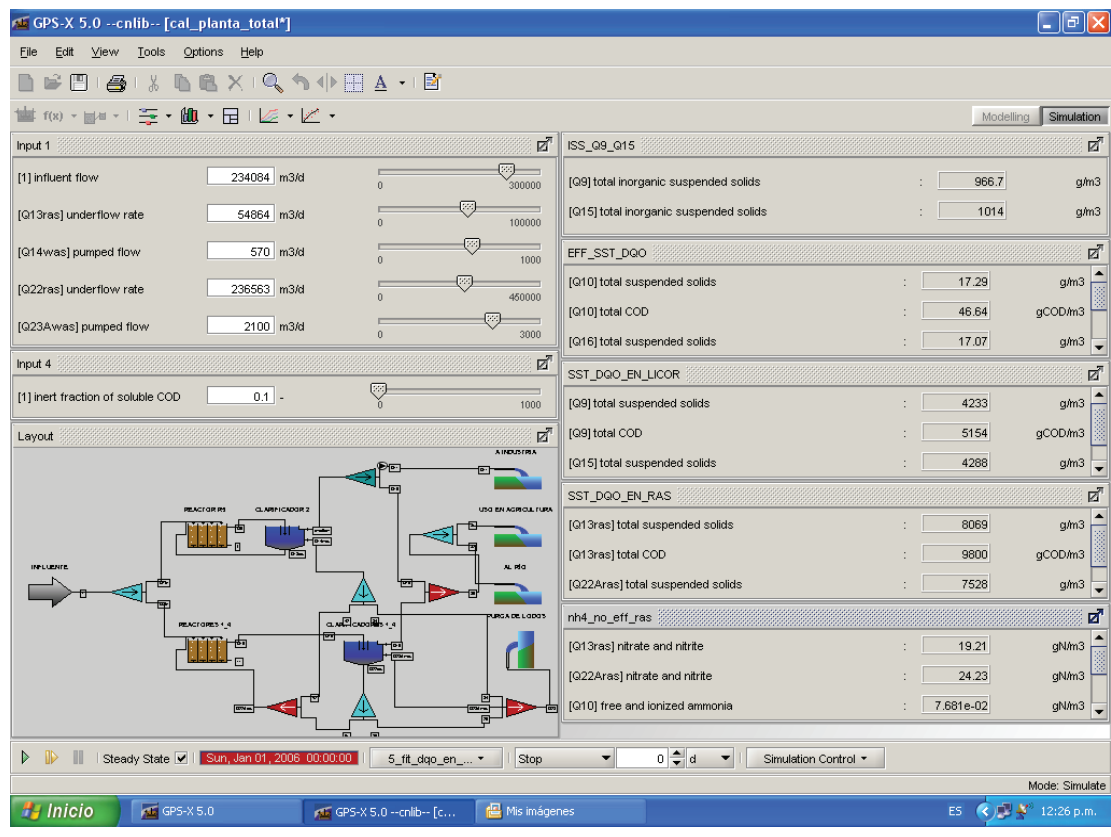

Figura 9. Calibración de la PTAR total. Escenario de simulación con GPS-X
Tabla 6. Comparación de resultados de ISS y SST calibrados versus campaña de muestreo

\begin{tabular}{ccc}
\hline Flujo & $\begin{array}{c}\text { Resultados de calibración } \\
\text { con GPS-X }\left(\mathrm{g} / \mathrm{m}^{3}\right)\end{array}$ & $\begin{array}{c}\text { Resultados de campaña } \\
\mathrm{g} / \mathrm{m}^{3}\end{array}$ \\
\hline \multicolumn{3}{c}{ ISS } \\
\hline $\mathrm{Q}_{9}$ & 966.7 & 963.0 \\
$\mathrm{Q}_{15}$ & 1014 & 1013.0 \\
\hline \multicolumn{3}{c}{} \\
\hline $\mathrm{Q}_{10}$ & 17.29 & SST \\
$\mathrm{Q}_{16}$ & 17.07 & 14.6 \\
$\mathrm{Q}_{9}$ & 4233.0 & 11.5 \\
$\mathrm{Q}_{15}$ & 4288.0 & 4156.0 \\
$\mathrm{Q}_{13}$ & 8069.0 & 4318.0 \\
$\mathrm{Q}_{22}$ & 7528.0 & 7790.0 \\
\hline
\end{tabular}

de recirculación $Q_{13}$ y $Q_{22}$ regresaron al valor original mostrado en la tabla 5 y figuras 5 y 6 . Con estos reajustes finales, también se alcanzaron los niveles óptimos de operación de la PTAR en cuanto a remoción de materia orgánica (DQO) y nitrógeno $\left(\mathrm{N}^{-\mathrm{NO}_{3}^{-}}\right.$y $\left.\mathrm{N}-\mathrm{NH}_{3}\right)$. A manera de comparación, en la tabla 6 se muestran los resultados obtenidos de los ISS y SST de la calibración con el software GPS-X y los resultados obtenidos durante la campaña intensiva de muestreo.

\section{Conclusiones}

Aún cuando el monitoreo de flujos no se alcanzó a realizar en la mayoría de los canales y tuberías de la PTAR, sí se logró calcular los flujos no conocidos a través de un balance de gastos. Por otra parte, para cerrar satis- factoriamente los balances de masa totales, se utilizaron los flujos $Q_{5}, Q_{14}$ y $Q_{23}$ que son las purgas de lodos, mientras que para cerrar los balances alrededor de las unidades individuales del proceso de lodos activados se consideraron dos opciones:

a) incrementar el flujo del RAS y disminuir el flujo del influente a reactores $\left(Q_{7}\right.$ y $\left.Q_{8}\right)$, y viceversa,

b) reducir el RAS y aumentar el flujo del influente a reactores $\left(Q_{7}\right.$ y $\left.Q_{8}\right)$.

La opción más factible fue la del caso a) pero solamente disminuyendo el flujo del influente a reactores, ya que al incrementar el flujo del RAS, el resultado fue un escenario fuera de la realidad.

El uso del balance de materia resultó una ventaja muy importante para detectar el comportamiento de la planta antes de su calibración, así por ejemplo, se verificaron los caudales y la composición de las corrientes en cuanto a SST, ISS y $P_{\text {total }}$. También, para los escenarios de calibración de los ISS en los reactores con $\mathrm{Q}_{\mathrm{WAS}} \mathrm{y}$ la calibración de los SST en el RAS, se realizó lo mismo que lo ejecutado en la hoja de Excel, solo que en este caso se usó el simulador GPS-X. Esto indica que los resultados obtenidos en la hoja de Excel pueden servir como base y se pueden acoplar fácilmente para la calibración del modelo ASM1.

Finalmente la práctica del ajuste en la purga de lodos en conjunto con el cierre del balance de materia durante la calibración del modelo ASM1, representan una opción aceptable para optimizar la producción de lodos, 
remoción de materia orgánica y nitrógeno. Con ello inclusive, también se pueden simular escenarios de ampliación de la planta de tratamiento de aguas residuales.

\section{Agradecimientos}

Los autores agradecen a Servicios de Agua y Drenaje de Monterrey (SADM) por permitir el uso de sus instalaciones para la realización de este proyecto de investigación, así como a la UNESCO-IHE y a la UAEM por su financiamiento.

\section{Referencias}

Clesceri L.S., Greenberg A.E., Trussell R.R. Standard Methods for the Examination of Water and Wastewater, 17a ed., USA, APHAAWWA-WPCF, 1989.

Espinosa M.A., Flores N., Esparza M., Fall C. Efecto de la temperatura en la tasa de crecimiento y decaimiento heterotrófico en el rango de $20-32^{\circ} \mathrm{C}$ en un proceso de lodos activados. Revista Mexicana de Ingeniería Química, volumen 11 (número 2), 2012: 309-321.

Fall C. y Loaiza N.J. Design of a Tracer Test Experience and Dynamic Calibration of the Hydraulic Model for a Full-Scale Wastewater Treatment Plant by Use of AQUASIM. Water Environment Research, volumen 79 (número 8), 2007: 893-900.

Fall C., Espinosa M.A., Flores N., Van Loosdrecht M.C.M., Hooijmans C.M. Stepwise Calibration of the Activated Sludge Model No. 1 at a Partially Denitrifying Large Wastewater Treatment Plant. Water Environment Research, volumen 83 (número 11), 2011: 2036-2048.

Gürkan S., Stijn W.H., Dirk J.W., Van Griensven A., Vanrolleghem P.A.A Critical Comparison of Systematic Calibration Protocols for Activated Sludge Models: A SWOT Analysis. Water Research, volumen 39 (número 12), 2005: 2459-2474.

Henze M., Grady C.P.L., Gujer W., Marais G.V.R., Matsuo T. Activated Sludge Model No. 1. England, International Association on Water Pollution Research and Control, 1987, pp. 37.
Henze M., Gujer W., Mino T., Van Loosdrecht, M.C.M. Activated Sludge Models ASM1, ASM2, ASM2d and ASM3, London UK, IWA Scientific and Technical Report 9, 2002, pp. 1-121.

Hulsbeek J.J.W., Kruit J., Roeleveld P.J., Van Loosdrecht M.C.M. A Practical Protocol for Dynamic Modelling of Activated Sludge Systems. Water Science and Technology, volumen 45 (número 6), 2002: 127-136.

Langergraber G., Rieger L., Winkler S., Alex, J., Wiese J., Owerdieck C., Ahnert M., Simon J., Maurer M. A Guideline for Simulation Studies of Wastewater Treatment Plants. Water Science and Technology, volumen 50 (número 7), 2004: 131-138.

Meijer S.C., Van Der Spoel H., Susanti S., Heijne J.J., Van Loosdrecht M.C. Error Diagnostics and Data Reconciliation for Activated Sludge Modelling Using Mass Balances. Water Science and Technology, volumen 45 (número 6), 2002: 145-156.

Petersen B., Gearney K., Henze M., Vanrolleghem P.A. Evaluation of an ASM1 Model Calibration Procedure on a Municipal-Industrial Wastewater Treatment Plant. Journal of Hydroinformatics, volumen 4, 2002: 15-38.

Water Environment Research Foundation (WERF). Methods for Wastewater Characterization in Activated Sludge Modelling, 1st ed., Alexandria, Virginia, WERF Publication No. 9 WWF3, 2003, pp. 596.

\section{Este artículo se cita:}

\section{Citación estilo Chicago}

Espinosa-Rodríguez, Miguel Angel, Cheikh Fall. Optimización de la producción de lodos en un sistema de lodos activados a través de la calibración del modelo ASM1. Ingeniería Investigación y Tecnología, XVI, 01 (2015): 93-104.

\section{Citación estilo ISO 690}

Espinosa-Rodríguez M.A., Fall- C. Optimización de la producción de lodos en un sistema de lodos activados a través de la calibración del modelo ASM1. Ingeniería Investigación y Tecnología, volumen XVI (número 1), enero-marzo 2015: 93-104.

\section{Semblanza de los autores}

Miguel Ángel Espinosa-Rodríguez. Ingeniero químico industrial con maestría en ingeniería ambiental y doctorado en ingeniería con énfasis en ciencias del agua. Profesor investigador del programa académico de ingeniería química del área de ciencias básicas e ingenierías en la Universidad Autónoma de Nayarit. Es coordinador del cuerpo académico de química ambiental y de la academia de ciencia e ingeniería ambiental. En 13 años de docencia e investigación, ha dirigido tesis de licenciatura y de maestría. Ha participado con ponencias en congresos nacionales e internacionales y ha publicado artículos de investigación.

Cheikh Fall. Ingeniero mecánico con maestría y doctorado en ingeniería química. Profesor-investigador del Centro Interamericano de Recursos del Agua (CIRA) dependiente de la Facultad de Ingeniería en la Universidad Autónoma del Estado de México. Docente de la maestría y doctorado en ingeniería con énfasis en ciencias del agua que se ofrece en el CIRA. Miembro del cuerpo académico de tratamiento de aguas y control de la contaminación. Ha dirigido tesis de licenciatura, maestría y doctorado, ha participado en ponencias en congresos nacionales e internacionales. Ha publicado artículos de investigación. 